\title{
Synthesis and antitumor efficacy of daunorubicin-loaded magnetic nanoparticles
}

\author{
This article was published in the following Dove Press journal: \\ International Journal of Nanomedicine \\ 21 January 20II \\ Number of times this article has been viewed
}

\author{
Jun Wang' \\ Baoan Chen' \\ Jian Chen' \\ Xiaohui Cai \\ Guohua Xia ${ }^{2}$ \\ Ran Liu' \\ Pingsheng Chen ${ }^{2}$ \\ Yu Zhang \\ Xuemei Wang ${ }^{3}$ \\ 'Department of Hematology and \\ Oncology, Zhongda Hospital, School \\ of Medicine, Southeast University, \\ Nanjing, China $210009 ;{ }^{2}$ Medical \\ School, Southeast University, \\ Nanjing; ${ }^{3}$ State Key Laboratory of \\ Bioelectronics, Southeast University, \\ Nanjing, China 210009
}

Correspondence: Baoan Chen Department of Hematology, The Affliated Zhongda Hospital, Southeast University, Nanjing 210009, People's Republic of China Tel +86258327 2006

Fax +8625832720II

Email cba8888@hotmail.com
Background: A promising approach to optimize the disposition of daunorubicin-loaded magnetic nanoparticles (DNR-MNPs) was developed to minimize serious side effects of systematic chemotherapy for cancer.

Methods: The physical properties of DNR-MNPs were investigated and their effect on leukemia cells in vitro was evaluated by a standard WST-1 cell proliferation assay. Furthermore, cell apoptosis and intracellular accumulation of DNR were determined by FACSCalibur flow cytometry.

Results: Our results showed that the majority of MNPs were spherical and their sizes were from 10 to $20 \mathrm{~nm}$. The average hydrodynamic diameter of DNR-MNPs in water was $94 \mathrm{~nm}$. The in vitro release data showed that the DNR-MNPs have excellent sustained release property. Proliferation of K562 cells was inhibited in a dose-dependent manner by DNR in solution (DNR-Sol) or by DNR-MNPs. The $\mathrm{IC}_{50}$ for DNR-MNPs was slightly higher than that for DNR-Sol. DNR-MNPs also induced less apoptosis in K562 cells than did DNR-Sol. Detection of fluorescence intensity of intracellular DNR demonstrated that DNR-MNPs could be taken up by K562 cells and persistently released DNR in cells.

Conclusion: Our study suggests that optimized DNR-MNPs formulation possesses sustained drug-release and favorable antitumor properties, which may be used as a conventional dosage form for antitumor therapy.

Keywords: daunorubicin, magnetic iron oxide nanoparticles, drug delivery system, target selection, K562 cells

\section{Introduction}

Chemotherapy is the foundation of treatment for the vast majority of cancers, including hematologic malignancies. Daunorubicin (DNR), one of the anthracycline antitumor agents, has been widely used in the treatment of acute myelocytic leukemia and acute lymphocytic leukemia. Its anticancer effect results from interference with DNA, reactive oxygen species, DNA topoisomerase I and II, metal ions, and induction of apoptosis. ${ }^{1}$ Nevertheless, the clinical use of DNR has been impeded by its serious side effects, including cardiotoxicity, myelosuppression, and oral ulcers. ${ }^{2}$ Nowadays, it is considered important to achieve maximum antitumor effects while minimizing undesirable side effects associated with high dosages of chemotherapy.

Nanotechnology holds tremendous promise for the diagnosis and treatment of cancer. ${ }^{3,4}$ Recently, nanoparticle-based drug delivery systems have been used in an attempt to target specific delivery of drugs and reducing dose-limiting side effects, and these play an important role in raising target/nontarget concentration ratios, increasing drug residence at the target site, and improving cellular uptake and intracellular 
stability. ${ }^{5}$ Scientists have studied drug delivery systems for many years, and recent achievements are very promising. ${ }^{6-8}$ Drug delivery systems have been developed by incorporating drugs into biodegradable polymeric nanoparticles, liposomes, solid lipid nanoparticles, surfactant-modified or lipid-modified hydrogels, or complex nonviral gene transfection systems. ${ }^{9}$ Biocompatible and biodegradable superparamagnetic iron oxide nanoparticles (MNPs), which can be targeted to desired sites by using an external magnet field, lose their magnetic properties once the magnet is removed. ${ }^{10}$ They have been used widely in various biomedical applications, such as enhanced resolution magnetic resonance imaging, tissue repair, drug delivery, cellular/molecular tracking, cell separation, tissue targeting, and transfection. ${ }^{11}$ Additionally, MNPs can be loaded with drugs and escape from the macrophages when they are coated with a biocompatible polymer. ${ }^{12}$ In view of these features, they are excellent candidates for targeted drug delivery. However, the drug loaded on the surface of MNPs by mechanical absorption will be released rapidly after intravenous injection, causing very limited amounts of drug to arrive at target sites.

In order to minimize side effects and maximize the efficacy of DNR, we have developed an innovative DNRMNPs formulation for sustained release of DNR, in which the iron oxide core is firstly coated with oleic acid and DNR is stored there, then stabilized by Pluronic F-127 ${ }^{\circledR}$. The physical characteristics of DNR-MNPs, including morphology, particle size, zeta potential, drug-loading capacity and encapsulation efficiency, kinetics of DNR release from magnetic nanoparticles in vitro, and magnetic properties, were comprehensively analyzed. Furthermore, the cytotoxic effects of the DNR-MNPs formulation on leukemia cells and induction of apoptosis were observed, and the efficacy of drug delivery was further investigated.

\section{Materials and methods \\ Materials}

Iron chloride hexahydrate $\left(\mathrm{FeCl}_{3} \cdot 6 \mathrm{H}_{2} \mathrm{O}\right)$ and iron sulfate heptahydrate $\left(\mathrm{FeSO}_{4} \cdot 7 \mathrm{H}_{2} \mathrm{O}\right)$ were obtained from Sinopharm Chemical Reagent Co., Ltd (Shanghai, China). Ammonium hydroxide $\left(\mathrm{NH}_{4} \cdot \mathrm{OH}\right)$ and oleic acid were acquired from Shanghai Lingfeng Chemical Reagent Co Ltd (Shanghai, China). Daunorubicin hydrochloride $(\mathrm{DNR} \cdot \mathrm{HCl})$ was purchased from Jinan Huifengda Chemical Co., Ltd (Shandong, China), and its stock solution was freshly prepared and stored in the dark at $4^{\circ} \mathrm{C}$. Pluronic F-127 was obtained from Sigma-Aldrich (St. Louis, MO). The WST-1 cell proliferation and cytotoxicity assay kit was obtained from Beyotime
Institute of Biotechnology (Jiangsu, China). The Annexin V-FITC apoptosis detection kit was obtained from Nanjing KeyGen Biotech Co., Ltd (Nanjing, China). All reagents were of analytical grade.

\section{Preparation of magnetic nanoparticles}

Iron oxide nanoparticles were prepared by coprecipitation of Fe (III) and Fe (II) with ammonium hydroxide in a nitrogen environment. In brief, ferric chloride hexahydrate $26 \mathrm{~g}$ and ferrous sulfate heptahydrate $18 \mathrm{~g}$ were dissolved in $50 \mathrm{~mL}$ of deionized water and heated to $70^{\circ} \mathrm{C}$. Then $50 \mathrm{~mL}$ of ammonium hydroxide (25\%) and $5 \mathrm{~g}$ of oleic acid were sequentially added. After 30 minutes, the temperature was increased to $80^{\circ} \mathrm{C}$ and maintained for one hour. The products, ie, oleic acid-coated iron oxide nanoparticles, were cooled to room temperature and extracted by a magnet. After being washed with ethanol and finally with deionized water, the precipitates were lyophilized and stored at room temperature.

\section{Synthesis of daunorubicin-loaded magnetic nanoparticles}

The formulation was developed as previously described. ${ }^{13}$ Briefly, $450 \mathrm{mg}$ of oleic acid-coated nanoparticles were dispersed in $45 \mathrm{~mL}$ of deionized water, and then $150 \mathrm{mg}$ of Pluronic F-127 was added. The mixtures were stirred on a magnetic stir plate overnight at room temperature and centrifuged at $1000 \mathrm{rpm}$ for 10 minutes at $10^{\circ} \mathrm{C}$ to obtain the oleic acid-coated and pluronic-stabilized iron oxide solution. The iron concentration in the aqueous dispersion of magnetic nanoparticles was detected by the 1, 10-phenanthroline colorimetric method. The hydrophobic DNR solution was then prepared by adding triethylamine $12 \mu \mathrm{L}$ to a DNR-methanolic solution $(5 \mathrm{mg} / \mathrm{mL}),{ }^{14}$ and $600 \mu \mathrm{L}$ of the solution obtained was added dropwise into an aqueous dispersion of MNPs (30 mg of nanoparticles in $7 \mathrm{~mL}$ of water) by violent magnetic force stirring for 16 hours to make the drug enter into the oleic acid layer. The unloaded free drug was separated from the MNPs by a magnet. Briefly, a magnet outside of the vial was used to attract the drug-loaded MNPs for five hours, and then the liquid was removed carefully without disturbing the MNPs. The drug-loaded MNPs were resuspended in deionized water and washed twice to obtain the synthesized DNR-MNPs.

\section{Morphology and appearance of MNPs}

The particle size and morphology of MNPs were observed by transmission electron microscopy (TEM; JEM-2100; JEOL, Tokyo, Japan). Briefly, the lyophilized MNPs were dispersed in deionized water with a water-bath sonicator for 
several minutes. A drop of MNPs solution was placed on a Formvar-coated copper TEM grid (150 mesh) and allowed to air dry for TEM observation.

\section{Fourier transfer infrared spectra}

The Fourier transfer infrared spectra of the specimens were recorded in the range of $400-4000 \mathrm{~cm}^{-1}$ using a Thermo Nicolet spectrometer (AVATAR 360 FT; Nicolet Instruments, Warwick, UK) at room temperature using the $\mathrm{KBr}$ pellet technique. The spectrum was recorded at room temperature, with a scanning speed of $20 \mathrm{~cm}^{-1}$ per minute and a spectral resolution of $4.0 \mathrm{~cm}^{-1}$.

\section{Crystallinity of MNPs by powder X-ray diffractometry}

The crystallinity of the formed MNPs was measured with a powder X-ray diffractometer (XD-3A; Shimadzu, Kyoto, Japan). An accelerating voltage of $25 \mathrm{kV}$ at $40 \mathrm{~mA}$ was used to produce vanadium-filtered $\mathrm{Cr} \mathrm{K} \alpha$ radiation between $20^{\circ}$ and $90^{\circ}$ at $27^{\circ} \mathrm{C}$.

\section{Size and zeta potential of DNR-MNPs}

The average hydrodynamic size of the DNR-MNPs was determined by dynamic laser scattering (Nano ZS90; Malvern Instruments, Westborough, MA). All measurements were done with an angle detection of $90^{\circ}$ at $25^{\circ} \mathrm{C}$ after the dispersion was diluted to an appropriate volume with deionized water (refractive index $=1.330$, diluent viscosity $=0.890 \mathrm{cP}$ ). The zeta potential of the DNR-MNPs was measured at $22^{\circ} \mathrm{C}$ using a Zeta Plus zeta potential analyzer (Brookhaven Instruments, Holtsville, NY). The samples were diluted with deionized water and measured in automatic mode (voltage $4.00 \mathrm{Vs}$, wavelength $658 \mathrm{~nm}$ ).

\section{Detection of DNR loading by high-performance liquid chromatography}

Drug loading of the DNR-MNPs was detected by highperformance liquid chromatography. Less than $2 \mathrm{mg}$ of lyophilized DNR-MNPs were dispersed in $2 \mathrm{~mL}$ of methanolic solution in chloroform $(12.5 \% \mathrm{v} / \mathrm{v})$ by shaking at a speed of $100 \mathrm{rpm}$ for 24 hours and then centrifuged for 10 minutes at $16,000 \mathrm{rpm}$. For each sample, $100 \mu \mathrm{L}$ of supernatant was diluted in $2 \mathrm{~mL}$ of methanolic-chloroform solution. Analysis of the sample was performed using a high-performance liquid chromatography system (Shimadzu, Kyoto, Japan) with a Science C18 column (4.6-250 mm, $5 \mathrm{~mm}$; Shimadzu) and a VPODS C18 column (Shimadzu) at a detection wavelength of $480 \mathrm{~nm}$ and an excitation wavelength of $560 \mathrm{~nm}$, respectively.
The concentration of DNR from 0.005 to $10 \mu \mathrm{g} / \mathrm{mL}$ was used to construct a calibration curve, and the DNR-loading capacity and encapsulation efficiency of nanoparticles were calculated as $\mathrm{DL}=\mathrm{We} / \mathrm{Wp} \times 100 \%, \mathrm{EE}=\mathrm{We} / \mathrm{Wt} \times 100 \%$ (DL, drug loading capacity; EE, encapsulation efficiency; We, weight of DNR encapsulated in nanoparticles; $\mathrm{Wp}$, weight of drug-loading magnetic nanoparticles; and $\mathrm{Wt}$, total weight of DNR added).

\section{Kinetics of DNR release from magnetic nanoparticles}

To study the kinetics of DNR release from MNPs, lyophilized DNR-MNPs were dispersed in $2.5 \mathrm{~mL}$ of phosphatebuffered saline $(\mathrm{pH}=7.4$, containing $0.1 \% \mathrm{w} / \mathrm{v}$ Tween-80, Tween-phosphate-buffered saline), and then placed in a dialysis membrane bag with a molecular weight cutoff of 8,000-14,000 Da. The dialysis membrane bag was bathed in a tube containing the same volume of Tweenphosphate-buffered saline and the tube was placed in a shaker bath at $37^{\circ} \mathrm{C}$. The buffer was removed completely at different time intervals and complemented with fresh buffer. Tween- 80 was added to keep sink conditions in the release experiments. The amount of DNR in buffer was analyzed by measuring the fluorescence intensity at $\lambda_{\mathrm{ex}}=458 \mathrm{~nm}$ and $\lambda_{\mathrm{em}}=591 \mathrm{~nm}$.

\section{Magnetization studies}

The magnetization of lyophilized DNR-MNPs was measured by a vibrating sample magnetometer integrated in a physical property measurement system (PPMS-9; Quantum Design, San Diego, CA) up to 50000 Oe at $300 \mathrm{~K}$.

\section{Cell culture}

Human chronic myelogenous leukemia cells (K562 cells) were donated by the Institute of Hematology at the Chinese Academy of Medical Sciences. Cells were maintained in RPMI 1640 medium containing 10\% heat-inactivated newborn bovine serum, $100 \mathrm{U} / \mathrm{mL}$ penicillin, and $100 \mu \mathrm{g} / \mathrm{mL}$ streptomycin at $37^{\circ} \mathrm{C}$ in a humidified $5 \% \mathrm{CO}_{2}$ atmosphere, and passaged once every $2-3$ days.

\section{In vitro cytotoxicity assays}

Cell viability was determined using a standard WST-1 cell proliferation assay. In brief, K562 cells were seeded in 96-well plates $(20,000$ cells per well), and administered with different concentrations of DNR in solution (DNRSol) or DNR-MNPs after four hours. The doses of DNR incorporated in MNPs were equal to the corresponding 
concentration of DNR-Sol. The cells were treated with RPMI 1640 medium and MNPs without drug as controls for DNR-Sol and DNR-MNPs, respectively. After being incubated for 48 hours, $20 \mu \mathrm{L}$ of WST-1 were added to each well and cultured for another four hours. The absorbance at $450 \mathrm{~nm}$ was determined using a microplate reader (Thermo Scientific Multiskan MK3, Boston, MA) and the viability was calculated as $\mathrm{OD}_{\text {treated cells }} / \mathrm{OD}_{\text {control cells }} \times 100 \%$. Finally, dose-effect curves were made and $\mathrm{IC}_{50}$ values were calculated.

\section{Flow cytometric apoptosis assay}

Based on the $\mathrm{IC}_{50}$ values of the WST-1 assay, $0.9 \mu \mathrm{g} / \mathrm{mL}$ DNR was adopted to test cell apoptosis by FACSCalibur flow cytometry (Becton Dickinson, Franklin Lakes, NJ). Briefly, $4 \times 10^{5} \mathrm{~K} 562$ cells were exposed to DNR or DNR-MNPs for 48 hours and, after being washed with phosphate-buffered saline twice, the cells were suspended with $500 \mu \mathrm{L}$ of binding buffer and labeled with $5 \mu \mathrm{L}$ of Annexin V-FITC for 15 minutes at room temperature in the dark. Thereafter, cell apoptosis was determined by flow cytometry using CellQuest software at excitation and emission wavelengths of $488 \mathrm{~nm}$ and $575 \mathrm{~nm}$, respectively, over one hour.

\section{Fluorescence intensity of intracellular DNR}

After incubation for 48 hours as described earlier, K562 cells were collected, washed three times with phosphate-buffered saline, and suspended with $500 \mu \mathrm{L}$ phosphate-buffered saline. Intracellular accumulation of DNR was detected by flow cytometry. The relative fluorescence intensity was calculated as $\mathrm{FI}_{\text {treated cells }} / \mathrm{FI}_{\text {control cells }}$.

\section{Statistical analysis}

All experiments were performed three times in duplicate. Data were expressed as mean \pm standard deviation. Statistical analyses were performed using a parametric test (Student's $t$-test) by means of SPSS software (version 13.0; SPSS Inc, Chicago, IL). A value of $P<0.05$ denoted statistical significance.

\section{Results}

\section{Physical characteristics of magnetic nanoparticles}

The majority of oleic acid-pluronic-stabilized magnetic nanoparticles under TEM were spherical or nearly spherical, with particle sizes from 10 to $20 \mathrm{~nm}$. In local fields of TEM, clusters of nanoparticles resulting from dehydration were also observed (Figure 1).

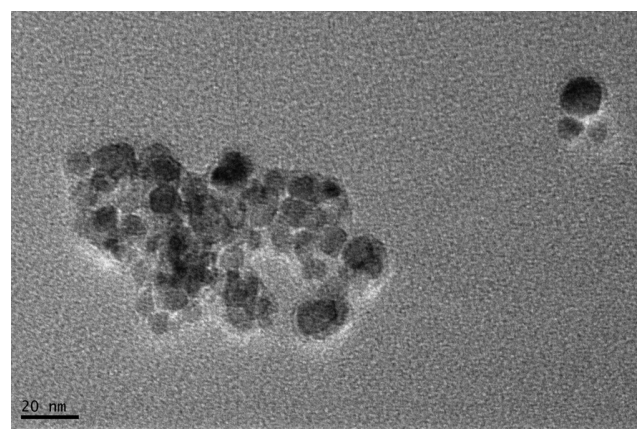

Figure I Oleic acid-pluronic-stabilized iron oxide nanoparticles under transmission electronic microscopy.

We examined the properties of pluronic-block copolymers as surface coatings for MNPs. Fourier transfer infrared spectra of MNPs formulation confirmed the anchoring of Pluronic F-127 onto the oleic acid-coated iron oxide core (Figure 2). The peaks appeared in the oleic acid-coated MNPs at 2920,2853 , and $1458 \mathrm{~cm}^{-1}$ owing to $-\mathrm{CH}_{2}-$ asymmetric or symmetric stretching and bending vibrations of the hydrophobic oleic acid chain. The hydrophilic carboxyl group on the pure oleic acid caused asymmetric vibration of the $\mathrm{C}=\mathrm{O}$ at $1708 \mathrm{~cm}^{-1},{ }^{15}$ but was absent in the spectra of oleic acid-coated MNPs, suggesting that oleic acid was chemisorbed as a carboxylate head group on the surface of the iron oxide nanoparticles (Figures 2A and 2C). Furthermore, the spectrum peaks of oleic acid-pluronic-stabilized MNPs at $1250-1000 \mathrm{~cm}^{-1}$ representing $-\mathrm{CH}_{2}$ - rocking and $\mathrm{C}-\mathrm{O}-\mathrm{C}$ stretch vibrations in polyethylene oxide (PEO)-polypropylene oxide (PPO) chains of Pluronic F-127 further confirmed the adsorption of each block copolymer to the surface of MNPs (Figures 2B and 2C).

To evaluate the crystal components, the diffraction peak of MNPs was analyzed by X-ray diffraction. Figure 3 indicated that the majority of the iron oxide were magnetite $\left(\mathrm{Fe}_{3} \mathrm{O}_{4}\right)$.

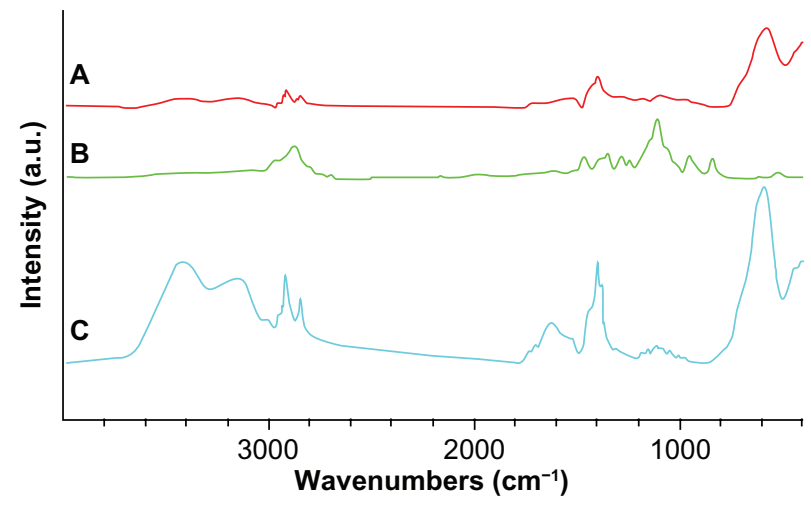

Figure 2 Fourier transfer infrared spectra by a Thermo Nicolet spectrometer. A) Oleic acid-coated iron oxide, B) pure Pluronic F-127, and C) oleic acid-pluronic stabilized iron oxide. 


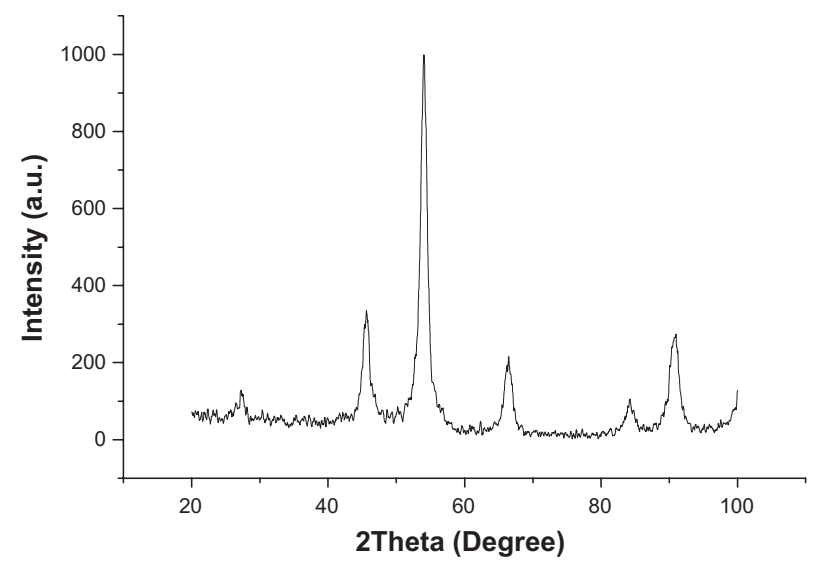

Figure 3 The crystallinity of pure $\mathrm{Fe}_{3} \mathrm{O}_{4}$ by $\mathrm{X}$-ray diffractometry.

The average hydrodynamic size of DNR-MNPs was $94.71 \mathrm{~nm}$ with a polydispersity index of 0.132 (Figure 4). Apparently, the hydrodynamic diameter detected by the particle size analyzer was due to the contribution of oleic acid and Pluronic F-127 associated with the nanoparticles and its hydration with water, which was larger than that of iron oxide observed under TEM. The zeta potential of DNR-MNPs in water was $-21.31 \pm 0.43 \mathrm{mV}$.

The drug-loading capacity and encapsulation efficiency were plotted in Table 1. The increase in the amount of drug was accompanied by an increase in drug-loading capacity. The encapsulation efficiency reached its peak when the added drug ratio was $10 \%$, suggesting that $10 \%$ of added drug was the optimal dosage.

According to the in vitro release data (Figure 5), about $20 \%$ of the cumulative drug release was in the first two days and $42 \%$ was in five days. After 25 days, the total drug release w $69 \%$, indicating that the DNR-MNPs had excellent sustained-release properties.

The hysteresis loops of DNR-MNPs were measured by a vibrating sample magnetometer in the maximum applied

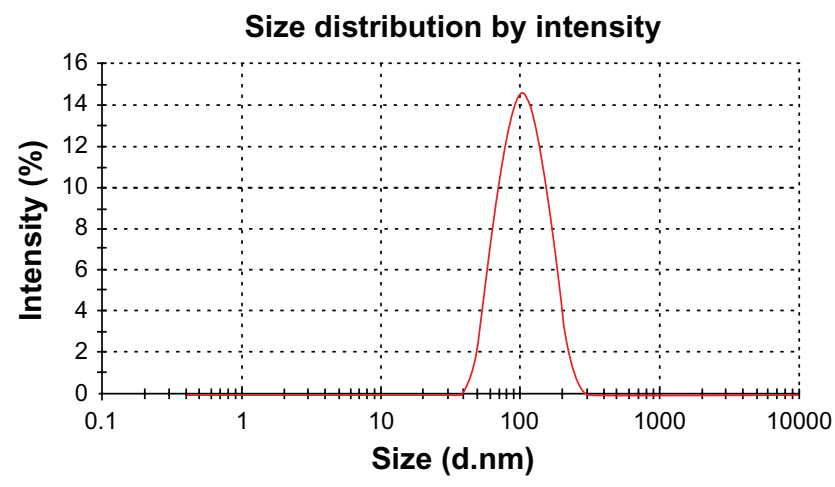

Figure 4 Hydrodynamic particle size distribution of daunorubicin-loaded magnetic nanoparticles in water measured by Zetasizer Nano ZS90 particle size analyzer.
Table I Daunorubicin loading in magnetic nanoparticles (mean \pm standard deviation, $\mathrm{n}=3$ )

\begin{tabular}{lcl}
\hline $\begin{array}{l}\text { Added } \\
(\% \mathrm{w} / \mathrm{w})\end{array}$ & $\begin{array}{l}\text { Loaded } \\
(\% \mathrm{w} / \mathrm{w})\end{array}$ & $\begin{array}{l}\text { Encapsulation } \\
\text { efficiency }(\%)\end{array}$ \\
\hline 5 & $2.07 \pm 0.11$ & 41.4 \\
10 & $7.62 \pm 0.47$ & 76.2 \\
20 & $11.49 \pm 0.68$ & 59.5 \\
\hline
\end{tabular}

field of $0 \mathrm{kOe}$ at room temperature (Figure 6). The saturation magnetization of the DNR-MNPs was $49.35 \mathrm{emu} / \mathrm{g}$, and coercivity $\mathrm{Hc}$ was $7.6 \mathrm{Oe}$ at room temperature. The samples showed typical superparamagnetic behavior at room temperature.

\section{Cytotoxicity of $\mathrm{K} 562$ cells}

The dose-dependent curve was calculated when K562 cells had been exposed to DNR-Sol or DNR-MNPs for 48 hours (Figure 7). Loss of cell viability was notable with increasing doses of both DNR-Sol and DNR-MNPs. Compared with DNR-Sol, the $\mathrm{IC}_{50}$ value for DNR-MNPs was slightly higher $\left(\mathrm{IC}_{50 / \mathrm{DNR}-\mathrm{MNPs}}=2.28 \pm 0.63 \mu \mathrm{g} / \mathrm{mL}, \mathrm{IC}_{50 / \mathrm{DNR}-\mathrm{Sol}}=0.86 \pm\right.$ $0.02 \mu \mathrm{g} / \mathrm{mL}, P<0.05)$. The MNPs alone had no obvious cytotoxic effect on K562 cells, and even the cell viability was still above $80 \%$ when the concentration of MNPs reached 100 $\mu \mathrm{g} / \mathrm{mL}$ after treatment for 48 hours (Figure 8).

\section{Apoptosis of $\mathrm{K} 562$ cells}

The percentages of apoptosis in K562 cells treated with DNR-Sol and DNR-MNPs were 50.07\% $\pm 7.39 \%$ and $35.27 \% \pm 5.27 \%$, respectively (Figure 9), suggesting that DNR-MNPs had a slightly lower ability to induce apoptosis of K562 cells when compared with DNR-Sol $(P<0.05)$.

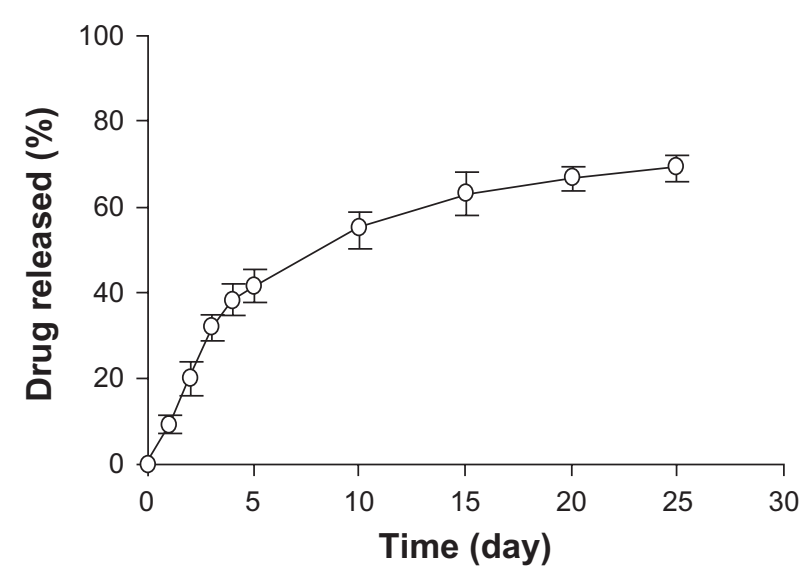

Figure 5 Release of daunorubicin from magnetic nanoparticles in vitro. Note: Data are mean \pm standard deviation $(n=3)$. 


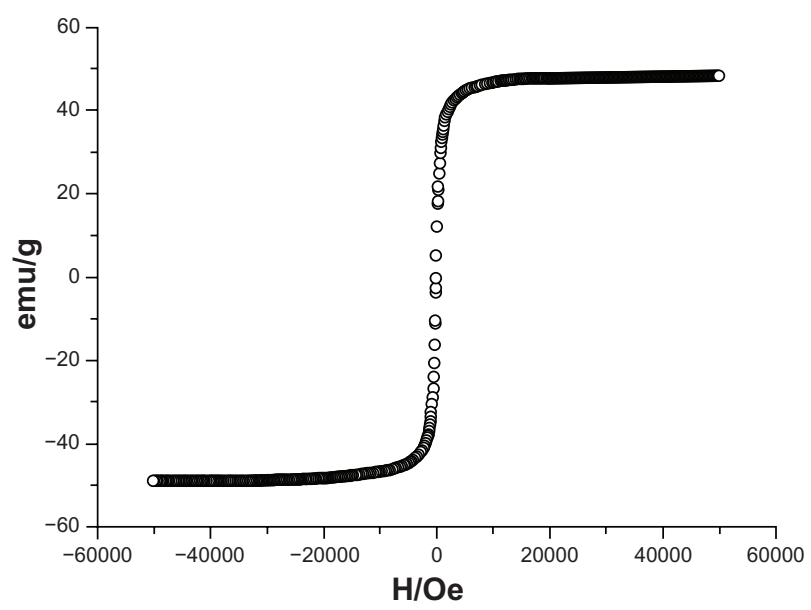

Figure 6 Magnetization as a function of field daunorubicin-loaded magnetic nanoparticles measured at $300 \mathrm{~K}$.

\section{Fluorescence intensity of intracellular DNR}

Due to the autofluorescence feature of DNR, intracellular DNR in K562 cells could be detected by flow cytometry. After incubation for 48 hours, the relative fluorescence intensity of intracellular DNR in cells treated with DNRSol and DNR-MNPs were $10.78 \pm 2.53$ and $10.79 \pm 1.59$, respectively. There was no statistically difference between them $(P>0.05$, Figure 10$)$.

\section{Discussion}

Chemotherapy has become one of the main strategies to treat most malignant tumors. However, there are some serious issues associated with systemic chemotherapy, including drug redistribution in the body and the high doses needed to

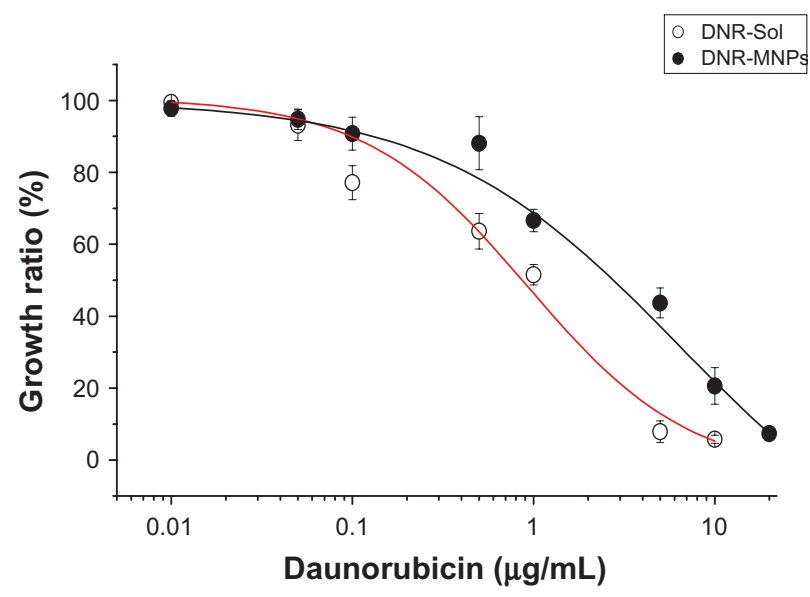

Figure 7 Cytotoxicity of $\mathrm{K} 562$ cells treated with different concentrations of DNRSol and DNR-MNPs by WST-I assay.

Note: Data are mean \pm standard deviation $(n=3)$.

Abbreviations: DNR-Sol, daunorubicin in solution; DNR-MNPs, daunorubicinloaded magnetic nanoparticles.

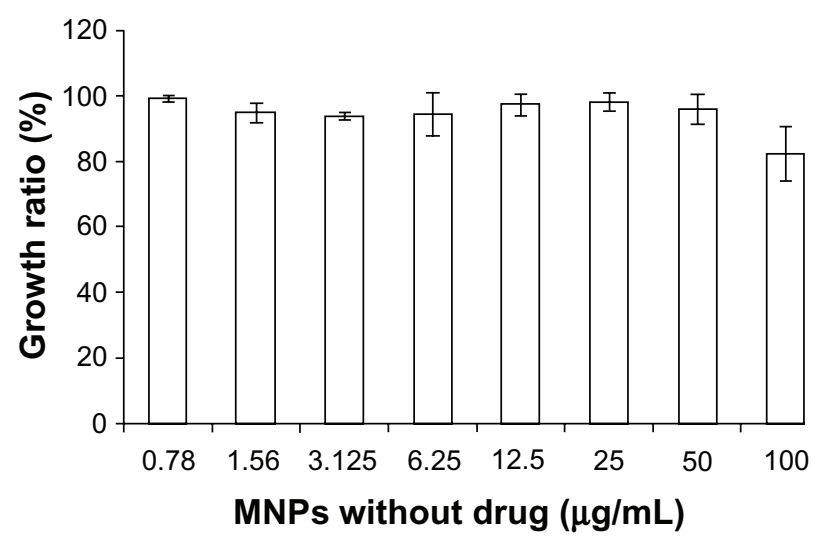

Figure 8 Cytotoxic effect of magnetic nanoparticles without drug on $\mathrm{K} 562$ cells. Note: Data are mean \pm standard deviation $(n=3)$.

Abbreviation: MNPs, magnetic nanoparticles.

reach target tissues in an effective concentration due to lack of site specificity. Because of toxicity to sensitive normal cells, nonspecific side effects emerge, including liver or kidney damage, bone marrow suppression, gastrointestinal reactions, and hair loss. Nanoparticle drug delivery systems have shown a potential to enhance the accumulation of anticancer agents in tumor cells. ${ }^{16}$ Optimization of these carriers continues today with the two objectives of reducing the amount of systemic distribution of the cytotoxic drug, thus reducing the associated side effects, and reducing the dosage required by more efficient localized targeting of the drug. ${ }^{17}$

Unlike normal tissues, cancer tissues usually consist of leaky blood vessels. Faced with fast-growing tumors, neovascularization is frequently defective and coupled with poor lymphatic drainage, which allows enhanced permeation and retention of therapeutic nanodrugs (enhanced permeability and retention effect). ${ }^{18-21}$ Therefore, it is possible to exploit nanoparticles as drug vehicles in tumor therapy because of their active and passive accumulation in tumors after intravenous injection. In addition, nanodrugs penetrate into target cancer cells via antibody- or ligand-mediated endocytosis, which increases the selective delivery of antineoplastic drugs to cancer cells or cancer-associated tissues, such as tumor vasculature, and limits their accumulation in healthy tissues. Thus, improvement of patient tolerance to chemotherapy, elevation of the clinical remission rate, and reversal of drug resistance in tumor cells can be realized. ${ }^{22-24}$

In this study, we have exploited iron oxide due to its biodegradability and biocompatibility as a nanocarrier system to transport DNR to the target site where it can exert its antitumor effects. In our DNR-MNPs formulation, iron oxide cores are coated with oleic acid and then modified by hydrophilic polymers to improve aqueous dispersion. Various polymers, such as polyvinylpyrrolidone, polylactic-co-glycolic acid, 
A

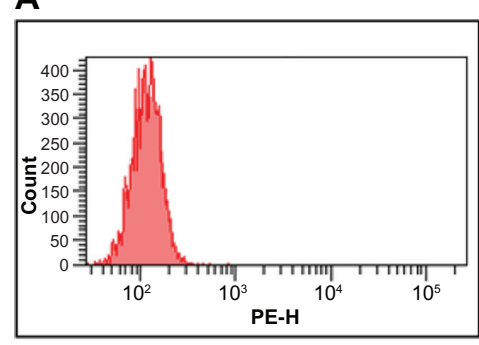

B

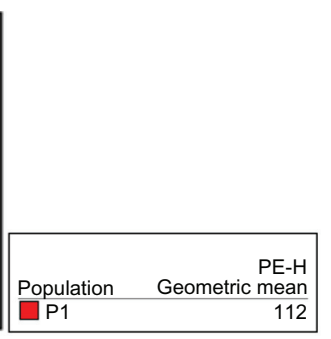

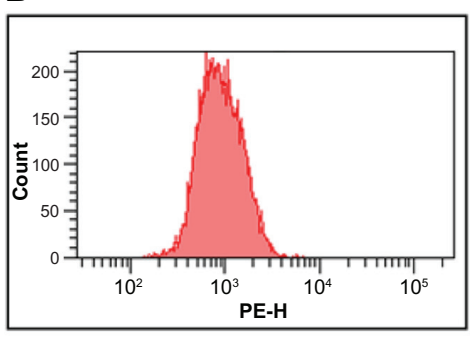

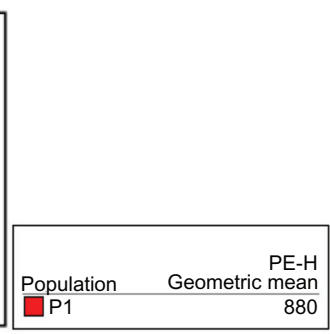

C

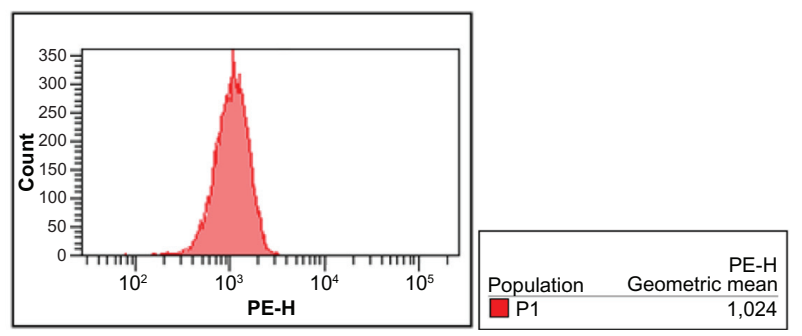

Figure 9 Cellular accumulation of DNR in K562 cells after treatment for 48 hours. A) control, B) DNR-Sol, and C) DNR-MNPs. Abbreviations: DNR, daunorubicin; DNR-Sol, daunorubicin in solution; DNR-MNPs, daunorubicin-loaded magnetic nanoparticles.

and polyethylene glycol, have been used as coating materials in aqueous suspension. Pluronics are amphiphilic synthetic polymers containing hydrophilic PEO blocks and hydrophobic PPO blocks arranged in a triblock structure, ie, PEOPPO-PEO.$^{25}$ The hydrophobic PPO of pluronics anchor onto the oleic acid shell and hydrophilic PEO have strong affinity for water molecules, which are responsible for the aqueous dispersibility of the formulation. Meanwhile, the hydrophilic surface is able to reduce opsonization and phagocytosis. ${ }^{26}$ We can utilize the magnetic property of iron oxide cores in the DNR-MNPs formulation and apply an external magnetic field to magnetize drug delivery system to target tissue. The oleic acid shell, acting as a drug depot, allows water-insoluble drugs, like DNR, to participate in oleic acid and persistently release DNR for a long period of time. Pluronic F-127 has the ability to form micelles spontaneously. However, because the concentration used in our experiment is much lower than the critical micelle concentration $(20 \mathrm{mg} / \mathrm{mL}),{ }^{27}$ our formulation is different from that of micelles described previously. ${ }^{28,29}$ Toxicity testing of MNPs without drug-loading confirms that they are nontoxic and safe. Compared with that described in Jain's report, ${ }^{13}$ our formulation possesses slightly lower drug-loading capacity, which may be related to the significantly reduced particle size. Moghimi et a ${ }^{30}$ concluded that small size
A

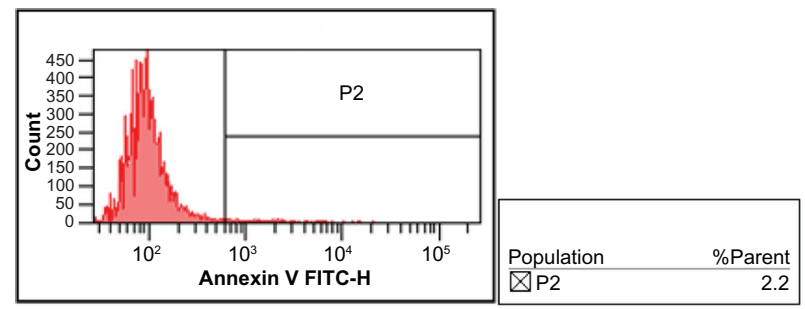

B

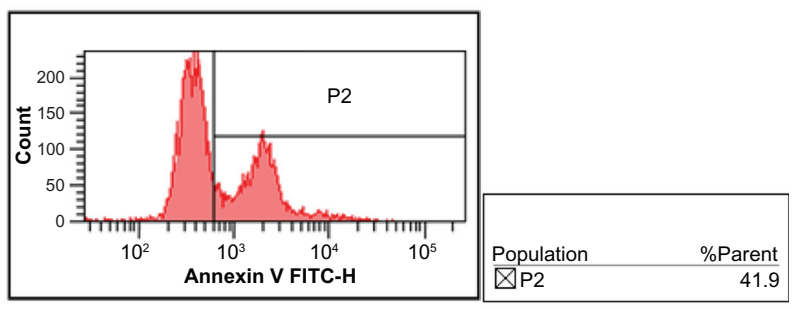

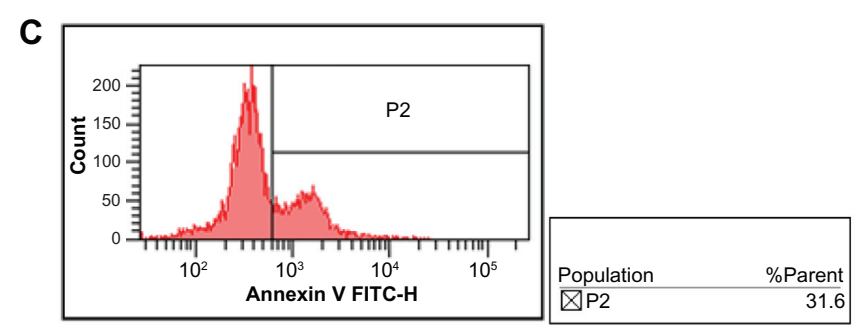

Figure 10 Effects of DNR-Sol and DNR-MNPs on apoptosis of K562 cells after treatment for 48 hours. A) control, B) DNR-Sol, and C) DNR-MNPs. Abbreviations: DNR-Sol, daunorubicin in solution; DNR-MNPs, daunorubicin-loaded magnetic nanoparticles; MNPs, magnetic nanoparticles. 
and high hydrophilicity of nanoparticles lead to decreasing opsonization. Gupta et $\mathrm{al}^{31}$ reported that sizes between 10 and $100 \mathrm{~nm}$ were the most effective for drug delivery because they could evade the reticulo-endothelial system (RES). Furthermore, our experimental model is in hematological malignancies, which is different to those used for solid tumors. According to the report of Moghimi et al, ${ }^{32}$ nanoparticles can penetrate into bone marrow easily when they are smaller than $100 \mathrm{~nm}$. Consequently, it seemed very important to control the particle size of our formulation. According to measurements using the particle size analyzer, an average DNR-MNPs size of $94 \mathrm{~nm}$ meets our requirements. Previously we used albumin to incorporate MNPs and DNR, but we abandoned this because of the low drug-loading capacity (about 3\%-5\%), large particle size $(>200 \mathrm{~nm})$, difficulty in forming spheres, and poor dispersion in water (data not shown). Currently, our DNR-MNPs are easily dispersed in water, and the drug-loading capacity and encapsulation efficiency are $7.62 \%$ and $76.2 \%$, respectively. In vitro drug release shows a biphasic pattern consisting of an initial burst release followed by sustained slow release over a prolonged period of time of up to several weeks. The drug loaded in the MNPs diffuses out from the oleic acid shell driven by a concentration gradient during the first few days, and then releases in a slow and sustained manner probably because of the dissociation of the OA layer from the iron oxide core. During the synthesis process, the physical characteristics of iron oxide do not change and it still possesses excellent superparamagnetic ability at room temperature. In in vitro cytotoxicity assays, the efficacy of DNR-MNPs is a little lower than that of DNR-Sol. However, at the termination of our experiment at 48 hours, only about $20 \%$ of the loaded DNR was released in vitro owing to the sustained-release action.

It is well known that apoptosis is a major mechanism of cell death. Similar results are found for the percentage of apoptotic K562 cells induced by DNR-Sol and DNR-MNPs. In a previous study by our group, ${ }^{33,34}$ daunorubicin-loaded magnetic nanoparticles of $\mathrm{Fe}_{3} \mathrm{O}_{4}$ were more effective than daunorubicin alone in multidrug-resistant cells. Our present synthesis method is different to the previous one, which relied on mechanical absorption and polymerization, resulting in loose linkage of DNR and MNPs. With its excellent sustained-release properties, our DNR-MNPs formulation continued to release DNR after arriving at the target site without significantly elevating plasma DNR concentrations. The results of our flow cytometric assay demonstrate that there is no difference in the amount of DNR uptaken in K562 cells between the DNR-Sol group and DNR-MNPs groups. Only about $20 \%$ of DNR is released from the nanoparticles over two days, according to the results of our in vitro release studies, suggesting that DNR-MNPs are taken up by K562 cells and release DNR in cells in a sustained manner, other than cells ingest DNR released from nanoparticles extracellularly.

We conclude that our DNR-MNPs formulation is an effective drug delivery system for systemic administration. Furthermore, it decreases the side effects of high plasma DNR concentrations after intravenous injection effectively, prolongs the dosing interval, and improves patient compliance.

\section{Conclusion}

Our study demonstrates that the DNR-MNPs formulation has good aqueous dispersion, small particle size, high drug-loading capacity and encapsulation efficiency, and has the properties of sustained drug release and superparamagnetism. It is a promising systemic chemotherapy for leukemia and can be expanded for use on other cancers. Further research is needed to test the effects and pharmacokinetics of our DNR-MNPs formulation in vivo, and its precise mechanism of action.

\section{Acknowledgments}

This work was financially supported by National Key Basic Research Program 973 of China (No 2010CB732404), National High Technology Research and Development Program 863 of China (No 2007AA022007) and National Nature Science Foundation of People's Republic China (No 3074006230872970). We thank Dr Yu Zang at the Southeast University of China for technical assistance.

\section{Disclosure}

The authors report no conflicts of interest in this work.

\section{References}

1. Lotfi K, Zackrisson AL, Peterson C. Comparison of idarubicin and daunorubicin regarding intracellular uptake, induction of apoptosis, and resistance. Cancer Lett. 2002;178:141-149.

2. Lu H, Yuan GX, He QH. Rapid analysis of anthracycline antibiotics doxorubicin and daunorubicin by microchip capillary electrophoresis. Microchem J. 2009;92:170-173.

3. Torchilin VP. Targeted pharmaceutical nanocarriers for cancer therapy and imaging. AAPS J. 2007;9:E128-E147.

4. Duncan R. Polymer conjugates for drug targeting. From inspired to inspiration! J Drug Target. 2006;14:333-335.

5. Ganta S, Devalapally H, Shahiwala A, et al. A review of stimuli-responsive nanocarriers for drug and gene delivery. J Control Release. 2008;126: 187-204.

6. Alexiou C, Schmid RJ, Jurgons R, et al. Targeting cancer cells: Magnetic nanoparticles as drug carriers. Eur Biophys J. 2006;35:446-450.

7. Nobs L, Buchegger F, Curny R, et al. Current methods for attaching targeting ligands to liposomes and nanoparticles. J Pharm Sci. 2004;93: 1980-1992.

8. Allen TM. Ligand-targeted therapeutics in anticancer therapy. Nat Rev Cancer. 2002;2:750-763. 
9. Sitterberg J, Ozcetin A, Ehrhardt C, et al. Utilising atomic force microscopy for the characterisation of nanoscale drug delivery systems. Eur J Pharm Biopharm. 2010;74:2-13.

10. Elias A, Tsourkas A. Imaging circulating cells and lymphoid tissues with iron oxide nanoparticles. Hematology Am Soc Hematol Educ Program. 2009;720-726.

11. Mahmoudi M, Simchi A, Imani M, et al. Optimal design and characterization of superparamagnetic iron oxide nanoparticles coated with polyvinyl alcohol for targeted delivery and imaging. $J$ Phys Chem B. 2008;112:14470-14481.

12. Tartaj P, Serna CJ. Synthesis of monodisperse superparamagnetic Fe/silica nanospherical composites. J Am Chem Soc. 2003;125: 15754-15755.

13. Jain TK, Morales MA, Sahoo SK, et al. Iron oxide nanoparticles for sustained delivery of anticancer agents. Mol Pharm. 2005;2: 194-205.

14. Kohori F, Sakai K, Aoyagi T, et al. Control of adriamycin cytotoxic activity using thermally responsive polymeric micelles composed of poly (N-isopropylacrylamide-co-N, N-dimethylacrylamide)-bpoly (D L-lactide). Colloids Surf B Biointerfaces. 1999;16:195-205.

15. Yallapu MM, Foy SP, Jain TK, et al. PEG-functionalized magnetic nanoparticles for drug delivery and magnetic resonance imaging applications. Pharm Res. 2010;27:2283-2295.

16. Orive G, Gascon AR, Hernandez RM, et al. Techniques: New approaches to the delivery of biopharmaceuticals. Trends Pharm Sci. 2004;25:382-387.

17. Mahmoudi M, Sant S, Wang B, et al. Superparamagnetic iron oxide nanoparticles (SPIONs): Development, surface modification and applications in chemotherapy. Adv Drug Deliv Rev. 2010 May 6. [Epub ahead of print]

18. Sledge GW Jr, Miller KD. Exploiting the hallmarks of cancer: The future conquest of breast cancer. Eur J Cancer. 2003;39: $1668-1675$.

19. Allen TM, Cullis PR. Drug delivery systems: Entering the mainstream. Science. 2004;303:1818-1822.

20. Conti M, Tazzari V, Baccini C, Pertici G, Serino LP, de Giorgi U. Anticancer drug delivery with nanoparticles. In Vivo. 2006;20: 697-701.

21. Iyer AK, Khaled G, Fang J, et al. Exploiting the enhanced permeability and retention effect for tumor targeting. Drug Discov Today. 2006;11: 812-818.
22. Liu Y, Miyoshi H, Nakamura M. Nanomedicine for drug delivery and imaging: A promising avenue for cancer therapy and diagnosis using targeted functional nanoparticles. Int J Cancer. 2007;120:2527-2537.

23. Jain KK. Nanomedicine: Application of nanobiotechnology in medical practice. Med Princ Pract. 2008;17:89-101.

24. Allen TM. Ligand-targeted therapeutics in anticancer therapy. Nat Rev Cancer. 2002;2:750-763.

25. Kabanov AV, Alakhov VY. Pluronic block copolymers in drug delivery: From micellar nanocontainers to biological response modifiers. Crit Rev Ther Drug Carrier Syst. 2002;19:1-72.

26. Besheer A, Vogel J, Glanz D, et al. Characterization of PLGA nanospheres stabilized with amphiphilic polymers: Hydrophobically modified hydroxyethyl starch vs pluronics. Mol Pharm. 2009;6: $407-415$.

27. Desai PR, Jain NJ, Sharma RK, et al. Effect of additives on the micellization of PEO/PPO/PEO block copolymer F127 in aqueous solution. Colloid Surf. 2001;178:57-69.

28. Batrakova EV, Li S, Brynskikh AM. Effects of pluronic and doxorubicin on drug uptake, cellular metabolism, apoptosis and tumor inhibition in animal models of MDR cancers. J Control Release. 2010; 143:290-301.

29. Sharma AK, Zhang L, Li S, et al. Prevention of MDR development in leukemia cells by micelle-forming polymeric surfactant. J Control Release. 2008;131:220-227.

30. Moghimi SM, Hunter AC, Murray JC. Long-circulating and targetspecific nanoparticles: Theory to practice. Pharmacol Rev. 2001;53: 283-318.

31. Gupta AK, Gupta M. Synthesis and surface engineering of iron oxide nanoparticles for biomedical applications. Biomaterials. 2005;26: 3995-4021.

32. Moghimi SM, Illum L, Davis SS. Physiopathological and physicochemical considerations in targeting of colloids and drug carriers to the bone marrow. Crit Rev Ther Drug Carrier Sys. 1990;7:187-209.

33. Chen BA, Mao PP, Cheng J, et al. Reversal of multidrug resistance by magnetic $\mathrm{Fe}_{3} \mathrm{O}_{4}$ nanoparticle copolymerizating daunorubicin and MDR1 shRNA expression vector in leukemia cells. Int J Nanomedicine. 2010;5:437-444

34. Chen BA, Lai BB, Cheng J, et al. Daunorubicin-loaded magnetic nanoparticles of $\mathrm{Fe}_{3} \mathrm{O}_{4}$ overcome multidrug resistance and induce apoptosis of K562-n/VCR cells in vivo. Int J Nanomedicine. 2009;4: 201-208.
International Journal of Nanomedicine

\section{Publish your work in this journal}

The International Journal of Nanomedicine is an international, peerreviewed journal focusing on the application of nanotechnology in diagnostics, therapeutics, and drug delivery systems throughou the biomedical field. This journal is indexed on PubMed Central, MedLine, CAS, SciSearch $®$, Current Contents ${ } /$ Clinical Medicine,

\section{Dovepress}

Journal Citation Reports/Science Edition, EMBase, Scopus and the Elsevier Bibliographic databases. The manuscript management system is completely online and includes a very quick and fair peer-review system, which is all easy to use. Visit http://www.dovepress.com/ testimonials.php to read real quotes from published authors. 